Research Article

\title{
Modelling to Engineering Data Using a New Class of Continuous Models
}

\author{
I. Elbatal $(\mathbb{D})$ and Naif Alotaibi \\ Department of Mathematics and Statistics-College of Science, \\ Imam Mohammad Ibn Saud Islamic University (IMSIU), Saudi Arabia \\ Correspondence should be addressed to I. Elbatal; iielbatal@imamu.edu.sa
}

Received 6 October 2021; Accepted 30 October 2021; Published 24 November 2021

Academic Editor: Badr Saad. T. Alkaltani

Copyright (C) 2021 I. Elbatal and Naif Alotaibi. This is an open access article distributed under the Creative Commons Attribution License, which permits unrestricted use, distribution, and reproduction in any medium, provided the original work is properly cited.

\begin{abstract}
In this paper, a new flexible generator of continuous lifespan models referred to as the Topp-Leone Weibull G (TLWG) family is developed and studied. Several mathematical characteristics have been investigated. The new hazard rate of the new model can be "monotonically increasing," "monotonically decreasing," "bathtub," and "J shape." The Farlie Gumbel Morgenstern (FGM) and the modified FGM (MFGM) families and Clayton Copula (CCO) are used to describe and display simple type Copula. We discuss the estimation of the model parameters by the maximum likelihood (MLL) estimations. Simulations are carried out to show the consistency and efficiency of parameter estimates, and finally, real data sets are used to demonstrate the flexibility and potential usefulness of the proposed family of algorithms by using the TLW exponential model as example of the new suggested family.
\end{abstract}

\section{Introduction and Motivation}

There has already been a great emphasis on building more flexible distributions in the recent past. To simulate reallife data in various practical disciplines, including finance, engineering, medical sciences, biological research, environmental studies, and insurance, over the last few decades, a variety of $\mathrm{G}$ families of distributions has been constructed and researched. We have generated numerous kinds of distributions by generalizing $\mathrm{G}$ families. With these new families, at least one shape parameter is merged with the baseline one, allowing for greater versatility, for instance, the generalized transmuted exponentiated $G$ [1], Weibull (W) G (WG) [2], the Burr type X-G by [3], Type II half logistic $G$ [4], exponentiated transmuted $G$ by [5], a new compound $\mathrm{G}$ family [6], the beta W G by [7], the generalized odd W G [8], the transmuted W G by [9], a new W G [10], TL G [11], a special generalized mixture class of probabilistic models [12], sine Topp-Leone G [13], Type 2 power ToppLeone G [14], a new version of Power Topp-Leone G [15], and Type 2 generalized Topp-Leone G [16], among others.
According to [11], the cumulative distribution function (CDF) of the TL G (TLG) class could well be found with

$$
F_{\alpha}(z)=\left[1-\bar{G}_{\underline{\Phi}}^{2}(z)\right]^{\alpha}=G_{\underline{\Phi}}^{\alpha}(z)\left[2-G_{\underline{\Phi}}(z)\right]^{\alpha},
$$

where $G_{\Phi}(z)$ refers to the CDF of the baseline model, and the corresponding density function (PDF) of (1) can be derived as

$$
f_{\alpha}(z)=2 \alpha g_{\underline{\Phi}}(z)\left[1-\bar{G}_{\underline{\Phi}}^{2}(z)\right]^{\alpha-1} \bar{G}_{\underline{\Phi}}(z),
$$

where $G_{\Phi}(z)=d G_{\Phi}(z) / d z$ refers to the PDF of the baseline model. According to [2], the WG family's CDF may be computed via

$$
G_{\underline{\Phi}}(z)=G_{\beta}(z)=1-\exp \left[-O_{\underline{\Phi}}(z)^{\beta}\right]
$$

where $O_{\underline{\Phi}}(z)=G_{\underline{\Phi}}(z) / \bar{G}_{\underline{\Phi}}(z)$ and $\bar{G}_{\underline{\Phi}}(z)=1-G_{\underline{\Phi}}(z)$. Then, 
TABle 1: Special cases.

\begin{tabular}{|c|c|c|}
\hline Baseline & New model & Corresponding CDF \\
\hline E & TLWE & $\left\{1-\exp \left[-2(\exp (\theta z)-1)^{\beta}\right]\right\}^{\alpha}$ \\
\hline W & $T L W W$ & $1-\exp \left(-2\left\{\exp \left[(\theta z)^{b}\right]-1\right\}^{\beta}\right)$ \\
\hline $\mathrm{Lx}$ & TLWLX & $\left(1-\exp \left\{-2\left[\left[1+\left(\frac{z}{b}\right)\right]^{\theta}-1\right]\right\}\right)$ \\
\hline $\mathrm{BX}$ & $T L W B X$ & $1-\exp \mid-2\left(\left\{1-\exp \left[-\left(\frac{z}{\theta}\right)^{2}\right]\right\}-1\right)$ \\
\hline LL & $T L W L L$ & $\left\{1-\exp \left[-2\left(\frac{z}{\theta}\right)^{b / \beta}\right]\right.$ \\
\hline $\mathrm{L}$ & TLWL & $1-\exp \left\{-2\left[\left(\frac{1+\theta+\theta z}{1+\theta}\right)^{-1}-1\right]\right.$ \\
\hline
\end{tabular}

using (3) and (1), the CDF of the TL W G (TLWG) class may indeed be expressed via

$$
F_{\alpha, \beta, \underline{\Phi}}(z)=\left\{1-\exp \left[-2 O_{\underline{\Phi}}(z)^{\beta}\right]\right\}^{\alpha}
$$

The corresponding PDF is

$f_{\alpha, \beta, \underline{\Phi}}(z)=2 \alpha \beta g_{\underline{\Phi}}(z) \exp \left[-2 O_{\underline{\Phi}}(z)^{\beta}\right] \frac{G_{\underline{\Phi}}(z)^{\beta-1}}{\bar{G}_{\underline{\Phi}}(z)^{\beta+1}}\left\{1-\exp \left[-2 O_{\underline{\Phi}}(z)^{\beta}\right]\right\}^{\alpha-1}$

The hazard rate function (HRF) can be easily derived using $h_{\alpha, \beta, \Phi}(z)=f_{\alpha, \beta, \Phi}(z) /\left[1-F_{\alpha, \beta, \Phi}(z)\right]$. The function $h_{\alpha, \beta, \Phi}(z)$ is called the failure rate of $F_{\alpha, \beta, \Phi}(z)$ and often referred to as the rate function or the intensity function or failure rate or instantaneous failure rate; in actuarial mathematics, it is named the "force of mortality," and in demographic disciplines, it is called the "mortality rate." It represents the failure intensity of an $x$-year-old equipment. It denotes the likelihood of an operational item failing in the next time period or the likelihood of a failure in a tiny unit interval of time $(z, z+\Delta z]$ given that no failure has occurred in $[0, z]$ and satisfies $h_{\alpha, \beta, \underline{\Phi}}(z)>0$ and $\int_{0}^{\infty} h_{\alpha, \beta, \underline{\Phi}}(z$ )$d x=0$. The HRF is critical because it intuitively translates as the level of risk associated with an object that has lived to time $x$. In life (death) tables, $h_{\alpha, \beta, \Phi}(z)$ is approximated by the probability that a certain individual of age $x$ will die during the next year. Some notions of aging refer to the HR such as $h_{\alpha, \beta, \Phi}^{\prime}(z)>0$ means positive aging, $h_{\alpha, \beta, \Phi}^{\prime}(z)=$ 0 means no aging, and $h_{\alpha, \beta, \Phi}^{\prime}(z)<0$ means negative aging. The idea of aging in statistical lifetime and reliability analysis does not imply that the unit grows older in the sense of time. Rather, it is a concept associated with residual life.

\section{Important Expansions}

Take a look at the binomial series expansion provided as

$$
\left(1-\frac{a_{1}}{a_{2}}\right)^{a_{3}-1}=\left.\sum_{l=0}^{\infty}(-1)^{l}\left(\begin{array}{c}
a_{3}-1 \\
l
\end{array}\right)\left(\frac{a_{1}}{a_{2}}\right)^{l}\right|_{\left(b>0 \text { and }\left|a_{1} / a_{2}\right|<1\right)} .
$$

Then, the PDF in (5) can be expressed as

$f_{\alpha, \beta, \underline{\Phi}}(z)=2 \alpha \beta g_{\underline{\Phi}}(z) \frac{G_{\Phi}(z)^{\beta-1}}{\bar{G}_{\underline{\Phi}}(z)^{1+\beta}} \sum_{l=0}^{\infty}(-1)^{l}\left(\begin{array}{c}\alpha-1 \\ l\end{array}\right) \underbrace{\exp \left[-2(1+l) O_{\underline{\Phi}}(z)^{\beta}\right]}_{A(z, \beta, \underline{\Phi})}$.

Applying the power series expansion to $A(z)$, we have

$$
\mathrm{A}(\mathrm{z}, \beta, \underline{\Phi})=\sum_{\mathrm{l}=0}^{\infty} \frac{1}{1 !}[-2(1+1)]^{\mathrm{l}} \mathrm{O}_{\underline{\Phi}}(\mathrm{z})^{\beta \mathrm{l}}
$$

where $O_{\underline{\Phi}}(z)^{\beta d}=G_{\underline{\Phi}}(z)^{\beta d} / \bar{G}_{\underline{\Phi}}(z)^{\beta d}$. Then,

$$
f_{\alpha, \beta, \underline{\Phi}}(z)=2 \alpha \beta \sum_{l, d=0}^{\infty} \frac{1}{d !}(-1)^{l+d}[2(1+l)]^{d}\left(\begin{array}{c}
\alpha-1 \\
l
\end{array}\right) g_{\underline{\Phi}}(z) \frac{G_{\underline{\Phi}}(z)^{(1+d) \beta-1}}{\bar{G}_{\underline{\Phi}}(z)^{(1+d) \beta+1}},
$$

but $\bar{G}_{\underline{\Phi}}(z)^{-(1+d) \beta-1}=\sum_{k=0}^{\infty}\left(\begin{array}{c}\beta(d+1)+1 \\ k\end{array}\right) G_{\Phi}(z)^{k}$. Then, the $f_{\alpha, \beta, \underline{\Phi}}(z)$ can be written as

$$
f_{\alpha, \beta, \underline{\Phi}}(z)=\left.\sum_{l, d, k=0}^{\infty} v_{(l, d, k)} h_{\beta^{*}}(z)\right|_{\left(\beta^{*}=\beta(1+d)+k\right)},
$$



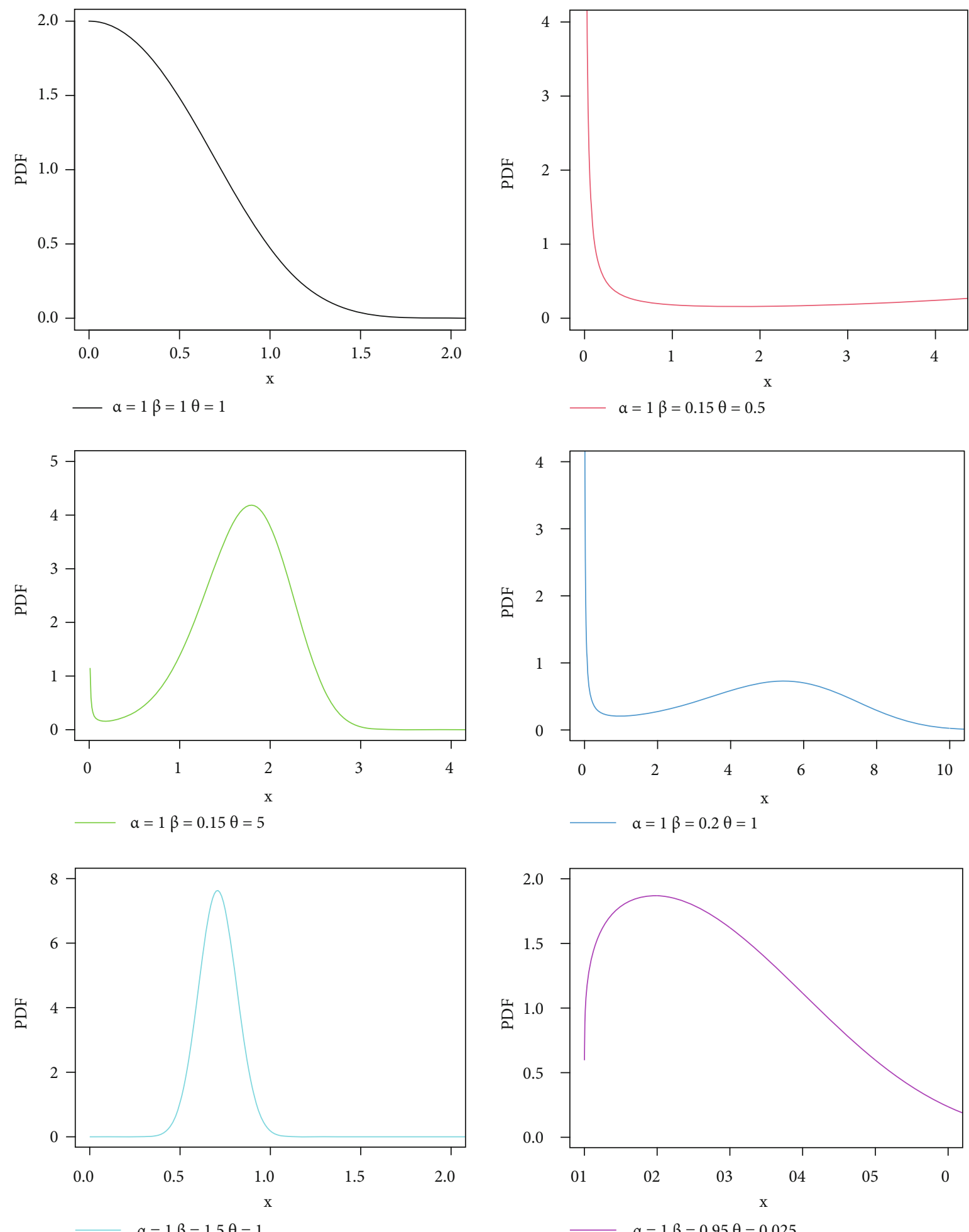

FIgure 1: Plots illustrating the PDF of the TLWE model. 

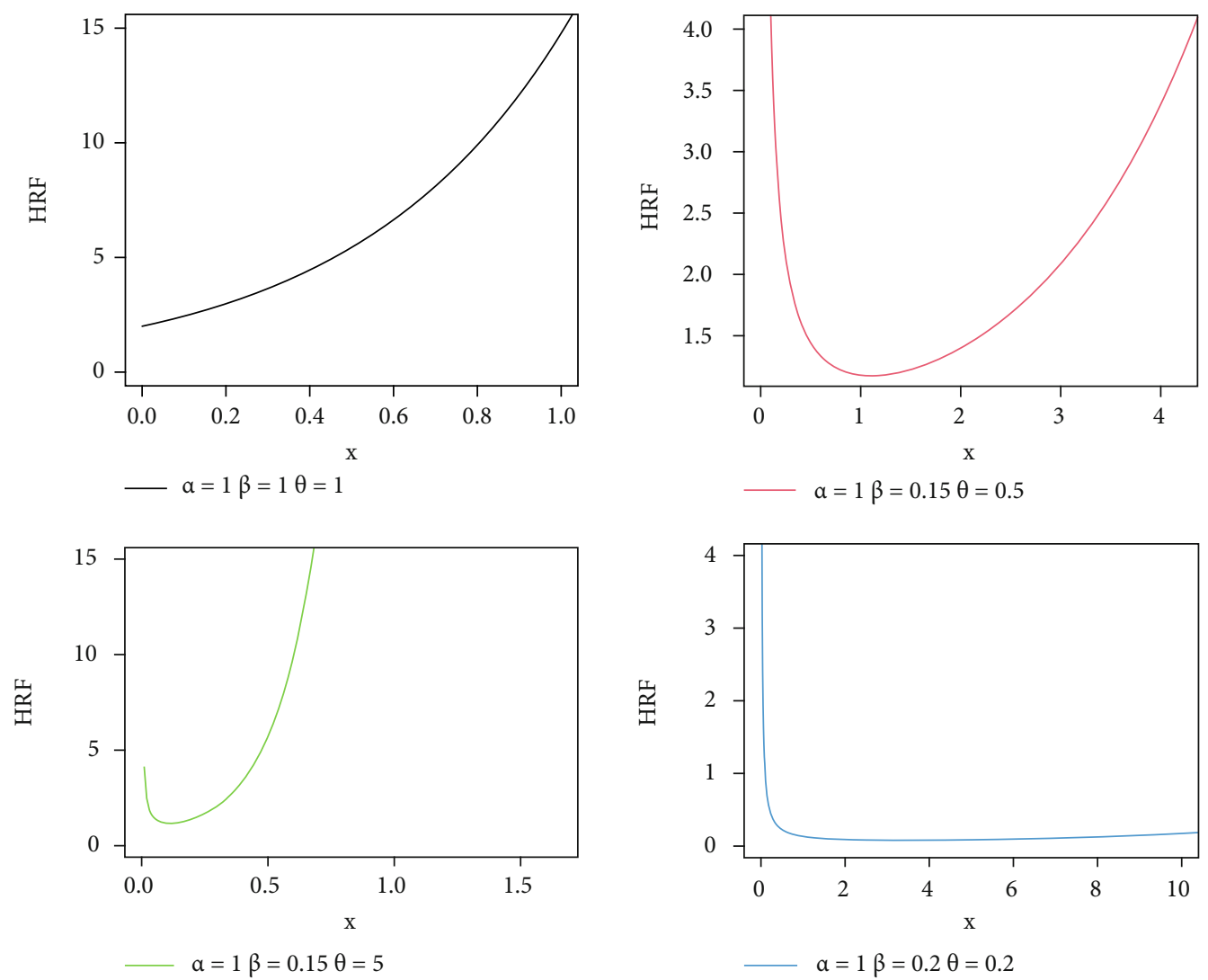

FiguRE 2: HRF graphs for the TLWE model.

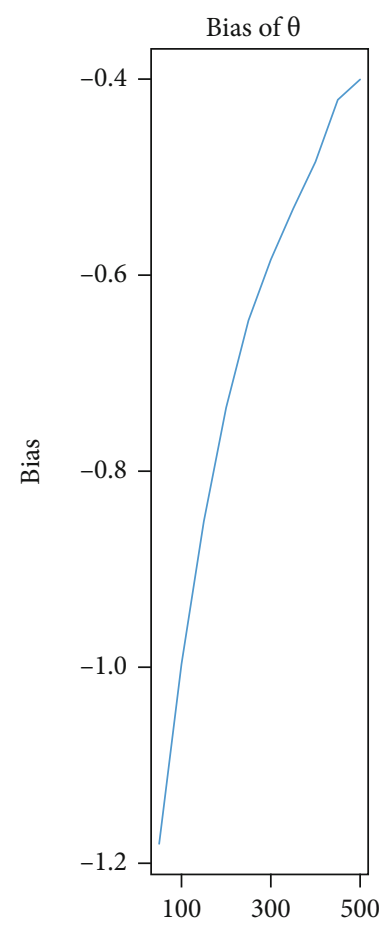

$\mathrm{n}$

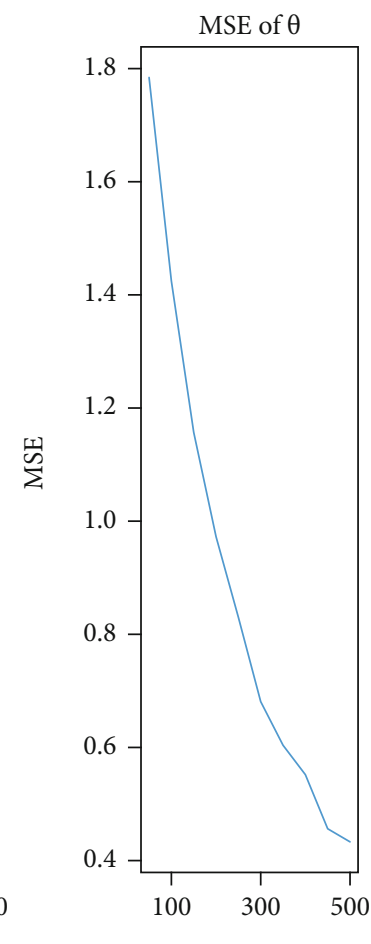

Figure 3: Biases and MSEs for the parameter $\alpha$.

where

$$
v_{(l, d, k)}=2 \alpha \beta \sum_{l, d, k=0}^{\infty}(-1)^{l+d}\left(\begin{array}{c}
\alpha-1 \\
l
\end{array}\right)\left(\begin{array}{c}
\beta(d+1)+1 \\
k
\end{array}\right) \frac{[2(l+1)]^{d}}{d ! \beta^{*}}
$$

and $h_{\beta^{*}}(z)=\beta^{*} g_{\Phi}(z) G_{\Phi}(z)^{\beta^{*}-1}$ depicts the PDF of the exponentiated $\mathrm{G}(\overline{\mathrm{ExG}})$ distribution with parameter $\beta^{*}$.

\section{Copula}

3.1. Via FGM Family. Starting with the joint CDF for the FGM family of random variables (RVrs) $\left(Z_{1}, Z_{2}\right)$ where $\left.F_{\lambda}(u, w)\right|_{(|\lambda| \leq 1)}=u w(1+\lambda \bar{u} \bar{w})$, let

$$
\begin{aligned}
& u=F_{\alpha_{1}, \beta_{1}, \underline{\Phi}}\left(z_{1}\right)=\left\{1-\exp \left[-2 O_{\underline{\Phi}}\left(z_{1}\right)^{\beta_{1}}\right]\right\}^{\alpha_{1}}, \\
& w=F_{\alpha_{2}, \beta_{2}, \underline{\Phi}}\left(z_{2}\right)=\left\{1-\exp \left[-2 O_{\underline{\Phi}}\left(z_{2}\right)^{\beta_{2}}\right]\right\}^{\alpha_{2}},
\end{aligned}
$$

where $O_{\Phi}\left(z_{1}\right)=G_{\Phi}\left(z_{1}\right) / \bar{G}_{\Phi}\left(z_{1}\right)$ and $O_{\Phi}\left(z_{2}\right)=G_{\Phi}\left(z_{2}\right) / \bar{G}_{\Phi}($ $\left.z_{2}\right)$; then, we have a $(5+\underline{\Phi})$ dimension parameter family

$$
\begin{aligned}
F_{\lambda}\left(z_{1}, z_{2}\right)= & \left\{1-\exp \left[-2 O_{\underline{\Phi}}\left(z_{1}\right)^{\beta_{1}}\right]\right\}^{\alpha_{1}}\left\{1-\exp \left[-2 O_{\underline{\Phi}}\left(z_{2}\right)^{\beta_{2}}\right]\right\}^{\alpha_{2}} \\
& \times\left\{1+\left[\begin{array}{l}
\left.\left.\lambda\left(1-\left\{1-\exp \left[-2 O_{\underline{\Phi}}\left(z_{1}\right)^{\beta_{1}}\right]\right\}^{\alpha_{1}}\right)\right]\right\} . \\
\left.\left.\times\left(1-\left\{1-\exp \left[-2 O_{\underline{\Phi}}\left(z_{2}\right)^{\beta_{2}}\right]\right\}^{\alpha_{2}}\right)\right]\right\}
\end{array}\right.\right.
\end{aligned}
$$






$\mathrm{n}$

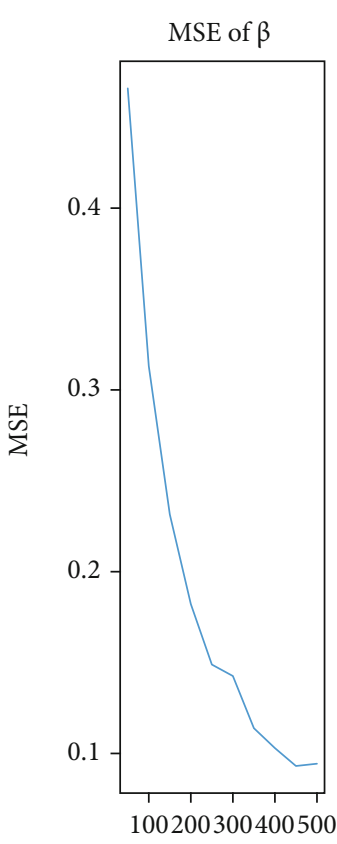

$\mathrm{n}$
FIGURE 4: Biases and MSEs for the parameter $\beta$.
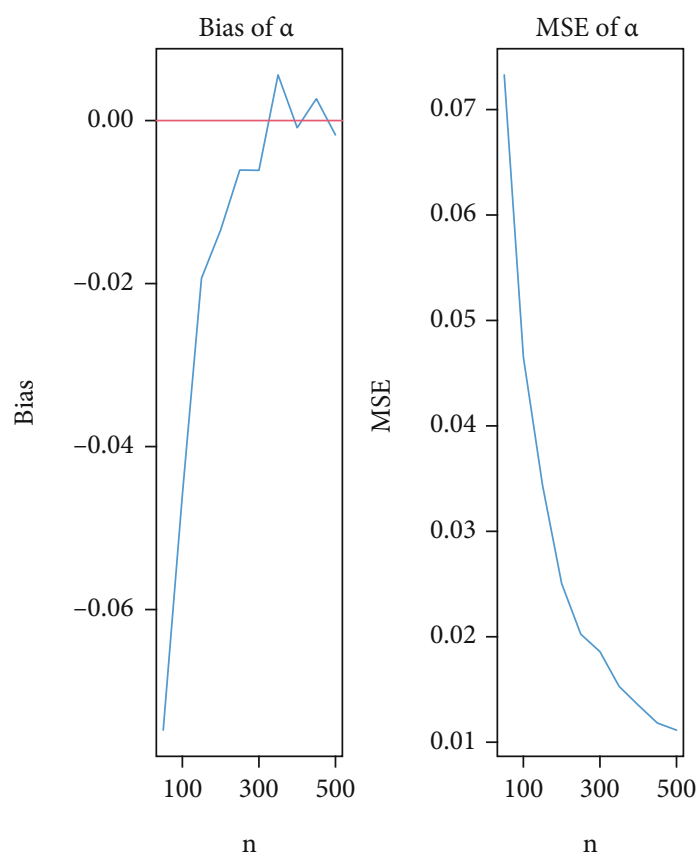

FIgURE 5: Biases and MSEs for the parameter $\theta$.

3.2. Via MFGM Copula. As an example, take the MFGM Copula (see [17-22])

$$
\left.C_{\tau}(u, v)\right|_{\tau \in[-1,1]}=u v[1+\tau \boldsymbol{\Phi}(u) \Psi(v)]=u v+\tau \dot{\Phi}(u) \dot{\Psi}(v),
$$

where $\dot{\boldsymbol{\Phi}}(u)=u \boldsymbol{\Phi}(u)$ and $\dot{\boldsymbol{\Psi}}(v)=v \boldsymbol{\Psi}(v)$. Where $\boldsymbol{\Phi}(u)$ and
$\Psi(v)$ are two absolutely continuous CDFs on $(0,1)$ where $\boldsymbol{\Phi}(0)=\boldsymbol{\Psi}(0)=\boldsymbol{\Phi}(1)=\boldsymbol{\Psi}(1)=0$, let

$$
\begin{aligned}
& \varepsilon=\ln f\left\{\frac{\partial \dot{\Phi}(u)}{\left.\partial u\right|_{C_{1}}}\right\}<0, \beta=\sup \left\{\frac{\partial \dot{\Phi}(u)}{\left.\partial u\right|_{C_{1}}}\right\}<0, \\
& \xi=\ln f\left\{\frac{\partial \dot{\Psi}(v)}{\left.\partial v\right|_{C_{2}}}\right\}>0, \eta=\sup \left\{\frac{\partial \dot{\Psi}(v)}{\left.\partial v\right|_{C_{2}}}\right\}>0 .
\end{aligned}
$$

Then, $\operatorname{mln}(\varepsilon \beta, \xi \eta) \geq 1$, where $\partial \dot{\boldsymbol{\Phi}}(u) / \partial u=\boldsymbol{\Phi}(u)+(u \partial \Phi$ $(u) / \partial u)$,

$$
\begin{aligned}
& C_{1}=\left\{u \mid u \in(0,1), \frac{\partial \dot{\Phi}(u)}{\partial u} \text { exists }\right\}, \\
& C_{2}=\left\{v \mid v \in(0,1), \frac{\partial \dot{\Psi}(v)}{\partial v} \text { exists }\right\} .
\end{aligned}
$$

\section{Type I MFGM:}

Consider $\boldsymbol{\Phi}(u)$ and $\boldsymbol{\Psi}(v)$ as defined above, then

$$
\begin{aligned}
C_{\tau}(u, v)= & \tau[\dot{\Phi}(u) \dot{\Psi}(v)]+\left(\left\{1-\exp \left[-2 O_{\underline{\Phi}}(u)^{\beta_{1}}\right]\right\}^{\alpha_{1}}\right. \\
& \left.\times\left\{1-\exp \left[-2 O_{\underline{\Phi}}(v)^{\beta_{2}}\right]\right\}^{\alpha_{2}}\right),
\end{aligned}
$$

where

$$
\begin{aligned}
& \dot{\Phi}(u)=u\left(1-\left\{1-\exp \left[-2 O_{\Phi}(u)^{\beta_{1}}\right]\right\}^{\alpha_{1}}\right), \\
& \dot{\Psi}(v)=v\left(1-\left\{1-\exp \left[-2 O_{\underline{\Phi}}(v)^{\beta_{2}}\right]\right\}^{\alpha_{2}}\right) .
\end{aligned}
$$

\section{Type II MFGM: \\ Let}

$$
\begin{aligned}
& \left.\boldsymbol{\Phi}(u)\right|_{\left(\tau_{1}>0\right)}=u^{\tau_{1}}(1-u)^{1-\tau_{1}}, \\
& \left.\Psi(v)\right|_{\left(\tau_{2}>0\right)}=v^{\tau_{2}}(1-v)^{1-\tau_{2}} .
\end{aligned}
$$

Then, the corresponding bivariate Copula can be derived directly from

$$
C_{\tau, \tau_{1}, \tau_{2}}(u, v)=u v\left[1+\tau u^{\tau_{1}} v^{\tau_{2}}(1-u)^{1-\tau_{1}}(1-v)^{1-\tau_{2}}\right] .
$$

\section{Type III MFGM:}

The CDF of the bivariate Type III MFGM model can be derived from

$$
C_{\tau}(u, w)=u F^{-1}(w)+w F^{-1}(u)-F^{-1}(u) F^{-1}(w),
$$



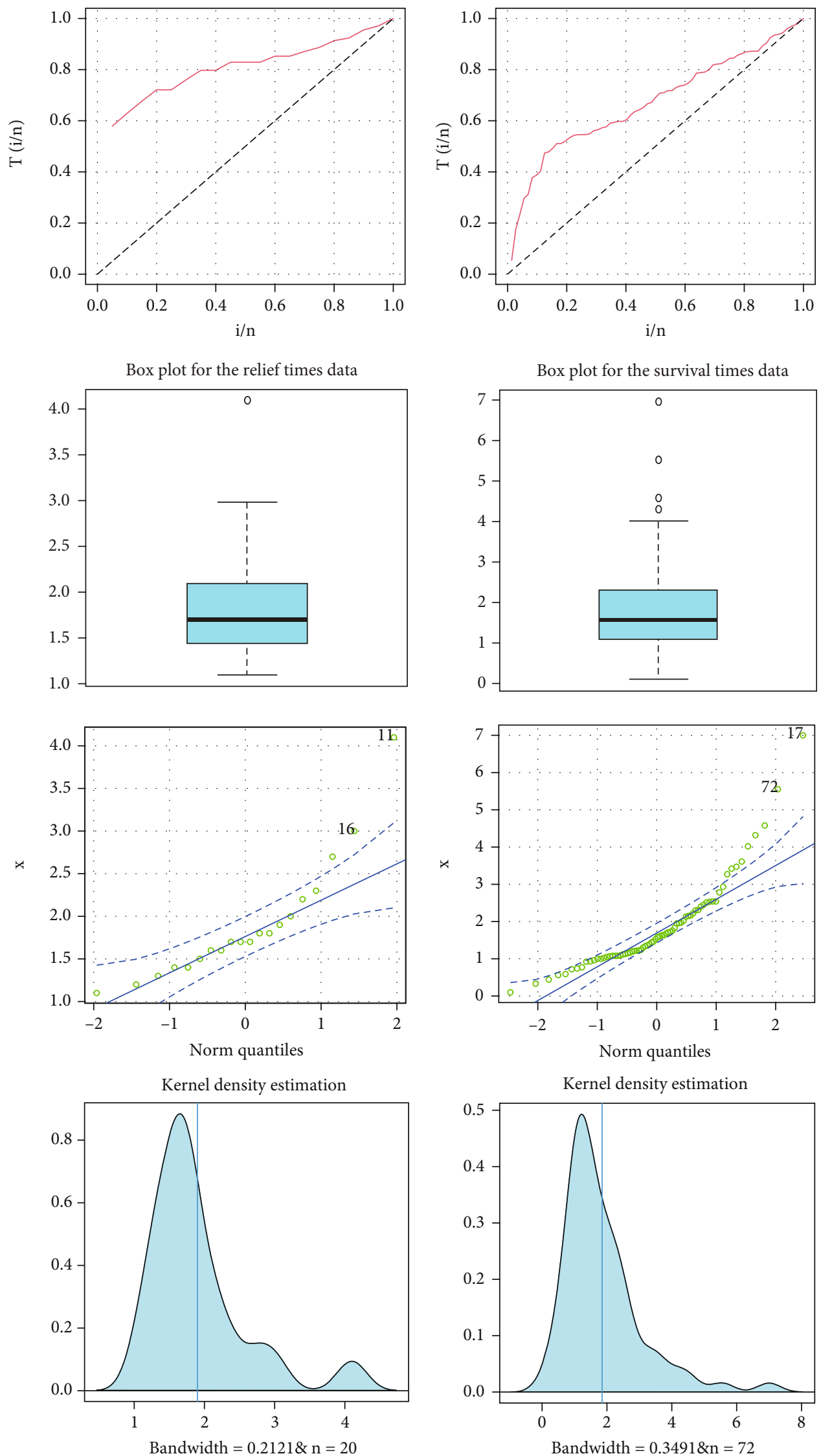

Figure 6: TTT plots, box plots, Q-Q plots, and KDE for the two real data sets. 
TABLE 2: MLLEs, SErs, and C.I. (in parentheses) values for the relief time data.

\begin{tabular}{|c|c|}
\hline Models & Estimates, SEs, and C.I.s \\
\hline \multirow{3}{*}{$E(\theta)$} & 0.5261 \\
\hline & $(0.1172)$ \\
\hline & $(0.3,0.8)$ \\
\hline \multirow{3}{*}{$\operatorname{ME}(\theta)$} & 0.950 \\
\hline & $(0.150)$ \\
\hline & $(0.7,1.2)$ \\
\hline \multirow{3}{*}{$\operatorname{LBHE}(\theta)$} & 0.5263 \\
\hline & $(0.118)$ \\
\hline & $(0.4,0.6)$ \\
\hline \multirow{3}{*}{$\operatorname{OLE}(\theta)$} & 0.6044 \\
\hline & $(0.0535)$ \\
\hline & $(0.5,0.7)$ \\
\hline \multirow{3}{*}{$\operatorname{BrXE}(a, \theta)$} & $1.1635,0.321$ \\
\hline & $(0.33),(0.03)$ \\
\hline & $(0.5,1.8),(0.26,0.4)$ \\
\hline \multirow{3}{*}{$\operatorname{MOE}(\alpha, \theta)$} & $54.47,2.32$ \\
\hline & $(35.58),(0.37)$ \\
\hline & $(0,124.2),(1.58,3.0)$ \\
\hline \multirow{3}{*}{$\operatorname{TLWE}(\alpha, \beta, \theta)$} & $8.03,1.58,3.15$ \\
\hline & $(4.22),(1.01),(0.025)$ \\
\hline & $(0,16.5),(0,3.6),(3.1,3.2)$ \\
\hline \multirow{3}{*}{$\mathrm{BE}(\alpha, \beta, \theta)$} & $81.633,0.542,3.514$ \\
\hline & (120.41), (0.327), (1.410) \\
\hline & $(0,317.63),(0,1.18),(0.75,6.3)$ \\
\hline \multirow{3}{*}{$\mathrm{KwE}(\alpha, \beta, \theta)$} & $83.756,0.568,3.330$ \\
\hline & (42.361), (0.326), (1.188) \\
\hline & $(0.7,167),(0,1.2),(1.00,5.7)$ \\
\hline \multirow{3}{*}{$\operatorname{GMOE}(\lambda, \alpha, \theta)$} & $0.519,89.462,3.169$ \\
\hline & (0.256), (66.278), (0.77) \\
\hline & $(0.02,1.02),(0,219.4),(1.66,4.7)$ \\
\hline \multirow{3}{*}{$\operatorname{KwMOE}(\alpha, \beta, \lambda, \theta)$} & $8.868,34.826,0.299,4.899$ \\
\hline & (9.15), (22.31), (0.24), (3.18) \\
\hline & $(10.9,46.8),(0,78.6),(0,0.76),(0,11)$ \\
\hline \multirow{3}{*}{$\operatorname{MOKwE}(\alpha, \beta, \lambda, \theta)$} & $0.133,33.232,0.571,1.669$ \\
\hline & $(0.332),(57.84),(0.72),(1.81)$ \\
\hline & $(0,0.8),(0,146.6),(0,2),(0,5.2)$ \\
\hline
\end{tabular}

where

$$
\begin{aligned}
& F^{-1}(u)=G^{-1}\left\{\frac{\left[-1 / 2 \log \left(1-u^{1 / \alpha_{1}}\right)\right]^{1 / \beta_{1}}}{1+\left[-1 / 2 \log \left(1-u^{1 / \alpha_{1}}\right)\right]^{1 / \beta_{1}}}\right\} \\
& F^{-1}(w)=G^{-1}\left\{\frac{\left[-1 / 2 \log \left(1-u^{1 / \alpha_{2}}\right)\right]^{1 / \beta_{2}}}{1+\left[-1 / 2 \log \left(1-u^{1 / \alpha_{2}}\right)\right]^{1 / \beta_{2}}}\right\}
\end{aligned}
$$

TABle 3: MLLEs, SErs, and C.I. (in parentheses) values for the

\begin{tabular}{|c|c|}
\hline Models & Estimates, SEs, and C.I.s \\
\hline \multirow{3}{*}{$E(b)$} & 0.540 \\
\hline & $(0.063)$ \\
\hline & $(0.4,0.7)$ \\
\hline \multirow{3}{*}{$\operatorname{OLE}(\theta)$} & 0.38145 \\
\hline & $(0.021)$ \\
\hline & $(0.3,0.4)$ \\
\hline \multirow{3}{*}{$\operatorname{ME}(\theta)$} & 0.9250 \\
\hline & $(0.080)$ \\
\hline & $(0.62,1.08)$ \\
\hline \multirow{3}{*}{$\operatorname{LBHE}(\theta)$} & 0.542 \\
\hline & $(0.06)$ \\
\hline & $(0.41,0.68)$ \\
\hline \multirow{3}{*}{$\operatorname{BrXE}(a, \theta)$} & $0.480,0.2060$ \\
\hline & $(0.061),(0.012)$ \\
\hline & $(0.4,0.5),(0.18,0.23)$ \\
\hline \multirow{3}{*}{$\operatorname{MOE}(\alpha, \theta)$} & $8.780,1.380$ \\
\hline & (3.555), (0.193) \\
\hline & $(1.81,15.74),(1.0,1.80)$ \\
\hline \multirow{3}{*}{$\operatorname{TLWE}(\alpha, \beta, \theta)$} & $3.225,1.55,0.018$ \\
\hline & (0.85), (0.25), (0.059) \\
\hline & $(1.5,4.9),(1,2),(0,0.136)$ \\
\hline \multirow{3}{*}{$\operatorname{GMOE}(\lambda, \alpha, \theta)$} & $0.179,47.635,4.470$ \\
\hline & (0.07), (44.901), (1.327) \\
\hline & $(0.04,0.3),(0,14),(2,7)$ \\
\hline \multirow{3}{*}{$\operatorname{KwE}(a, \beta, \theta)$} & $3.3039,1.101,1.038$ \\
\hline & (1.120), (0.763), (0.615) \\
\hline & $(1.12,5.53),(0,2.62),(0,2.24)$ \\
\hline \multirow{3}{*}{$\operatorname{MOKE}(\alpha, \beta, \lambda, \theta)$} & $0.008,2.716,1.986,0.099$ \\
\hline & $(0.002), 1.316),(0.784),(0.048)$ \\
\hline & $(0.004,0.010),(0.14,5),(0.4,4),(0,0.2)$ \\
\hline
\end{tabular}
survival time data.

3.3. Via CCO. The CCO is a weighted variant of the CCO, which has the following form:

$$
C(u, w)=\left[u^{-\tau}+v^{-\tau}-1\right]^{-\tau^{-1}}
$$

Then, setting

$$
\begin{aligned}
& u=u_{\alpha_{1}, \beta_{1}, \underline{\Phi}}\left(z_{1}\right)=\left\{1-\exp \left[-2 O_{\underline{\Phi}}(z)^{\beta_{1}}\right]\right\}^{\alpha_{1}}, \\
& w=w_{\alpha_{2}, \beta_{2}, \underline{\Phi}}\left(z_{2}\right)=\left\{1-\exp \left[-2 O_{\underline{\Phi}}(y)^{\beta_{2}}\right]\right\}^{\alpha_{2}},
\end{aligned}
$$


TABLE 4: Statistic for the relief time data.

\begin{tabular}{lcccc}
\hline Models & $(\mathrm{D} 2), \mathrm{D} 1$ & $C_{1}$ & $C_{2}$ & $C_{3}, C_{4}, C_{5}, C_{6}$ \\
\hline E & $(0.004), 0.4$ & 4.60 & 0.96 & $68.0,68.7,67.9,68.0$ \\
KwE & $(0.86), 0.14$ & 0.45 & 0.07 & $42.0,44.8,43.3,42.3$ \\
BrXE & $(0.17), 0.25$ & 1.33 & 0.24 & $48.1,50.1,49.0,48.5$ \\
MOE & $(0.55), 0.18$ & 0.80 & 0.14 & $43.5,45.5,44.2,43.9$ \\
GMOE & $(0.78), 0.15$ & 0.51 & 0.08 & $42.8,45.7,44.3,43.3$ \\
KMOE & $(0.86), 0.15$ & 1.08 & 0.19 & $43.0,46.8,45.6,43.6$ \\
MOKE & $(0.87), 0.14$ & 0.60 & 0.11 & $41.6,45.5,44.3,42.3$ \\
OLE & $(<0.1 \%), 0.9$ & 1.30 & 0.22 & $49.1,50.1,49.3,49.3$ \\
BE & $(0.80), 0.16$ & 0.70 & 0.12 & $43.5,46.5,44.9,44.0$ \\
ME & $(0.07), 0.32$ & 2.76 & 0.53 & $54.3,55.3,54.5,54.5$ \\
LBHE & $(<0.1 \%), 0.4$ & 0.62 & 0.11 & $67.7,68.7,67.9,67.8$ \\
TLWE & $(0.952), 0.10$ & 0.36 & 0.040 & $41.35,41.14,41.39,40.15$ \\
\hline
\end{tabular}

TABLE 5: Statistic for the survival time data.

\begin{tabular}{lcccc}
\hline Models & $(\mathrm{D} 2), \mathrm{D} 1$ & $C_{1}$ & $C_{2}$ & $C_{3}, C_{4}, C_{5}, C_{6}$ \\
\hline E & $(0.060), 0.27$ & 6.53 & 1.25 & $234.6,236.9,234.7,235.5$ \\
MOKE & $(0.440), 0.10$ & 0.79 & 0.12 & $209.4,218.6,210.0,213.0$ \\
OLE & $(<0.1 \%), 0.49$ & 1.94 & 0.33 & $229.1,231.4,229.2,230.0$ \\
ME & $(0.130), 0.14$ & 1.52 & 0.25 & $210.4,212.7,210.5,211.3$ \\
LBHE & $(<0.1 \%), 0.28$ & 0.79 & 0.19 & $235.0,237.0,235.0,236.0$ \\
GMOE & $(0.811), 0.09$ & 1.02 & 0.16 & $210.5,217.4,211.0,213.2$ \\
KwE & $(0.500), 0.09$ & 0.74 & 0.11 & $209.4,216.2,209.8,212.1$ \\
BrXE & $(0.002), 0.22$ & 2.90 & 0.52 & $235.3,239.9,235.5,237.1$ \\
MOE & $(0.430), 0.10$ & 1.20 & 0.17 & $210.4,215.0,210.5,212.2$ \\
TLWE & $(0.770), 0.066$ & 0.75 & 0.12 & $208.6,212.2,207.6,210.2$ \\
\hline
\end{tabular}

where

$$
\mathrm{O}_{\underline{\Phi}}(\mathrm{y})=\frac{\mathrm{G}_{\Phi}(\mathrm{y})}{\overline{\mathrm{G}}_{\underline{\Phi}}(\mathrm{y})} .
$$

Then,

$H(z, y)=\left(\left\{1-\exp \left[-2 O_{\underline{\Phi}}(z)^{\beta_{1}}\right]\right\}^{-\tau \alpha_{1}}+\left\{1-\exp \left[-2 O_{\underline{\Phi}}(y)^{\beta_{2}}\right]\right\}^{-\tau \alpha_{2}}-1\right)^{-\tau^{-1}}$.

A simple $d$-dimensional expansion of the above will be as follows:

$$
H\left(z_{1}, z_{2}, \cdots, z_{d}\right)=\left(\sum_{l=1}^{d}\left\{1-\exp \left[-2 O_{\Phi}\left(z_{l}\right)^{\beta_{l}}\right]\right\}^{-\tau \alpha_{l}}+1-d\right)^{-\tau^{-1}} .
$$

Recently, many new articles are allocated to study some of these types, see $[23,24]$.

\section{Structural Properties of the TLWG Family}

4.1. Quantile Function. The TLWG quantile function (QuF), say $z=Q(u)$, might be obtained by filliping (4); we have

$$
z=Q(u)=G^{-1}\left\{\frac{\left[-1 / 2 \log \left(1-u^{1 / \alpha}\right)\right]^{1 / \beta}}{1+\left[-1 / 2 \log \left(1-u^{1 / \alpha}\right)\right]^{1 / \beta}}\right\} .
$$
$(0,1)$

We can easily generate $z$ by taking $u$ as a uniform $\mathrm{RVr}$ in

4.2. Moments. The $r^{\text {th }}$ moment (MO) of TLWG could be acquired in the prescribed sequence:

$$
\mu_{r}^{\prime}=\int_{0}^{\infty} z^{r} f(z) d z=\sum_{l, d, k=0}^{\infty} v_{(l, d, k)} I_{(0, \infty)}\left(\beta^{*}\right),
$$

where $I_{(0, \infty)}\left(\beta^{*}\right)=\int_{0}^{\infty} z^{r} h_{\beta^{*}}(z) d z$ is the $r^{\text {th }}$ moment of the ExG model using parameter $\beta^{*}$.

4.3. Conditional Moments. The $s^{\text {th }}$ lower and upper incomplete MOs (ICMOs) of $Z$ characterized features $v_{s}(t)=E$ ( $\left.\left.Z^{s}\right|_{(Z<t)}\right)=\int_{0}^{t} z^{s} f(z) d z$ and $\zeta_{s}(t)=E\left(\left.Z^{s}\right|_{(Z>t)}\right)=\int_{t}^{\infty} z^{s} f(z) d z$, respectively, for just about every real $s>0$. The $s^{\text {th }}$ lower ICMO of TLWG is

$$
v_{s}(t)=\int_{0}^{t} z^{s} f(z) d z=\sum_{l, d, k=0}^{\infty} v_{(l, d, k)} I_{(0, t)}\left(\beta^{*}, s, t\right)
$$

where $I_{(0, t)}\left(\beta^{*}, s, t\right)=\int_{0}^{t} z^{s} h_{\beta^{*}}(z) d z$ is the $s^{\text {th }}$ lower ICMO of ExG model with exponential parameter $\beta^{*}$. Similarly, the $s^{\text {th }}$ upper ICMO of TLWG is

$$
\zeta_{s}(t)=\int_{t}^{\infty} z^{s} f(z) d z=\sum_{l, d, k=0}^{\infty} v_{(l, d, k)} I_{(t, \infty)}\left(\beta^{*}, s, t\right),
$$

where $I_{(t, \infty)}\left(\beta^{*}, s, t\right)=\int_{t}^{\infty} z^{s} h_{\beta^{*}}(z) d z$ is the $s^{\text {th }}$ upper ICM of ExG model with exponential parameter $\beta^{*}$.

4.4. Bonferroni and Lorenz Curves. A positive $\mathrm{RVr} \mathrm{Z}$ is described by the following Lorenz curve

$$
L(p)=\frac{1}{\mu} \int_{q}^{\infty} z f(z) d z=\frac{1}{\mu} \sum_{l, d, k=0}^{\infty} v_{(l, d, k)} I_{(q, \infty)}\left(\beta^{*}, 1, q\right),
$$

where $q=G^{-1}(p)$. Also, the Bonferroni curve is defined by

$$
B(p)=\frac{1}{p \mu} \int_{q}^{\infty} z f(z) d z=\sum_{l, d, k=0}^{\infty} v_{(l, d, k)} I_{(q, \infty)}\left(\beta^{*}, 1, q\right) .
$$

There are numerous uses for the Bonferroni curve in economics to analyze income and poverty, as well as dependability, medical, and insurance areas. 

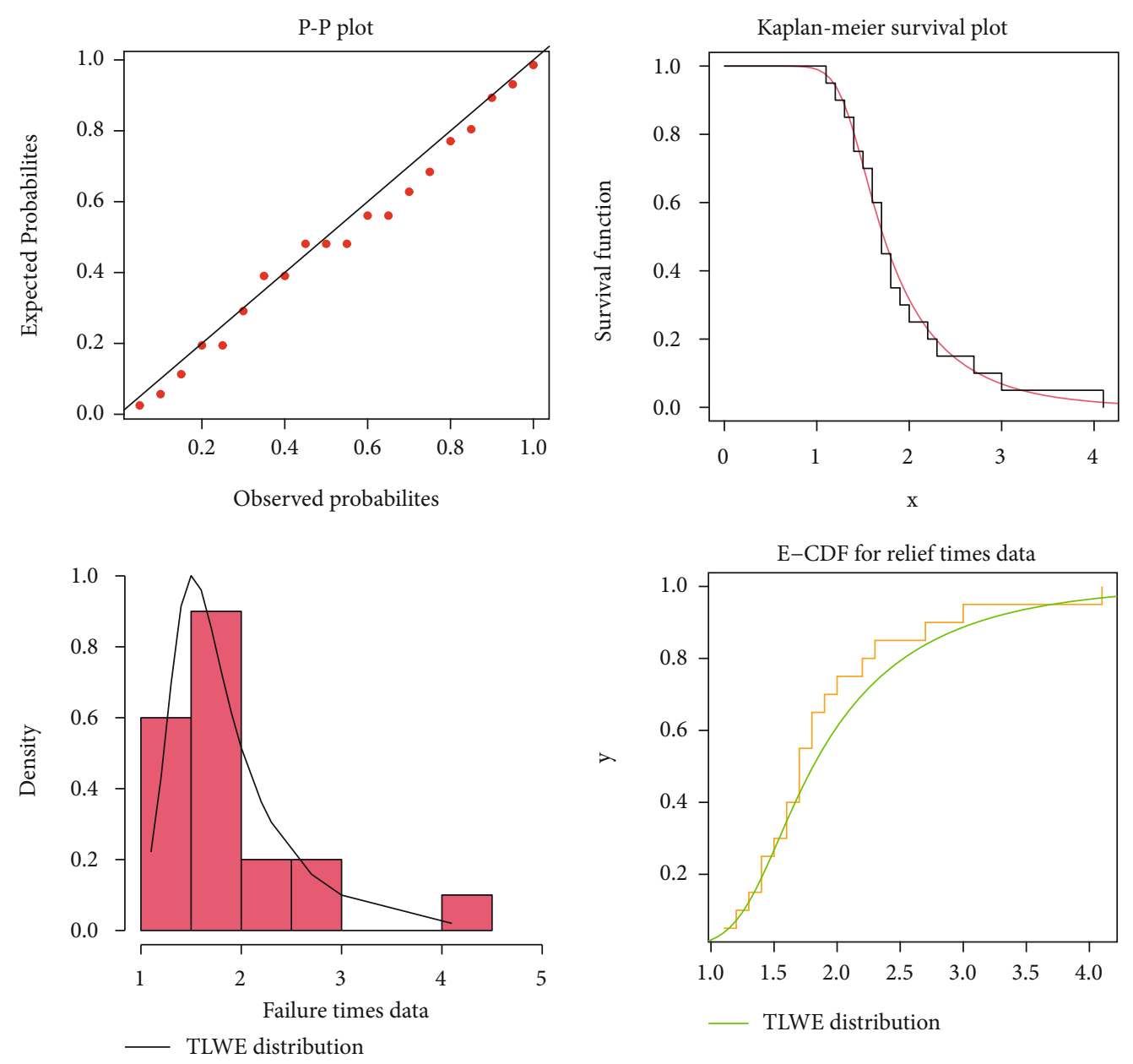

Figure 7: P-P, KMS plot, FPDF, and FCDF for the $1^{\text {st }}$ data.

\section{Special Cases}

In this part, we will look at various TLWG family-specific situations. We provide six TLWG family special models equivalent to the baseline exponential (E), Weibull (W), Lomax (Lx), Burr-X (BX), log-logistic (LL), and Lindley (L) distributions. The odd ratio $O_{\Phi}(z)$ of these baseline models along with the new models are listed in Table 1.

The above-mentioned PDF parameters are all positive actual numbers. Figure 1 shows graphs of the PDF of the TLWE model. Figure 2 depicts the TLWE model's HRF graphs. According to Figure 1, the new PDF can have a variety of useful forms. According to Figure 2, the new HRF can be increasing $(\alpha=\beta=\theta=1)$, bathtub $(\alpha=1, \beta=0.15, \theta=0.5$ ) , J shape $(\alpha=1, \beta=0.15, \theta=5)$, and decreasing $(\alpha=1, \beta=0.2, \theta=2)$.

\section{Maximum Likelihood (MLL) Estimation}

Suppose $z_{1}, \cdots, z_{n}$ be an $n^{\text {th }}$ random sample from the TLWG class provided via (5). Take $\underline{P}=(\alpha, \beta, \underline{\delta})^{T}$ become the vector of parameters. The total log-likelihood (LLL) function for $\underline{P}$ is

$$
\begin{aligned}
L_{n}(\underline{P})= & n \log (2 \beta \alpha)+\sum_{l=1}^{n} \log {g_{\underline{\Phi}}}_{l}\left(z_{l}\right)+(\beta-1) \sum_{l=1}^{n} \log G_{\underline{\Phi}}\left(z_{l}\right) \\
& -(\beta+1) \sum_{l=1}^{n} \log \bar{G}_{\underline{\Phi}}\left(z_{l}\right)-2 \sum_{l=1}^{n}\left[O_{\underline{\Phi}}\left(z_{l}\right)\right]^{\beta} \\
& +(\alpha-1) \sum_{l=1}^{n} \log \left(1-\exp \left\{-2\left[O_{\underline{\Phi}}\left(z_{l}\right)\right]^{\beta}\right\}\right),
\end{aligned}
$$

where $O_{\Phi}\left(z_{l}\right)=G_{\Phi}\left(z_{l}\right) / \bar{G}_{\Phi}\left(z_{l}\right)$. The LLL can really be optimized immediately employing SAS software or the R-language, or implicitly through solving nonlinear LL formulas acquired through differentiating (34). The score function's related components $U_{n}(\psi)=$ $\left(\partial L_{n}(\underline{P}) / \partial \alpha, \partial L_{n}(\underline{P}) / \partial \beta, \partial L_{n}(\underline{P}) / \partial \underline{\Phi}\right)^{T}$ are

$$
\frac{\partial L_{n}(\underline{P})}{\partial \alpha}=\frac{n}{\alpha}+\sum_{l=1}^{n} \log \left(1-\exp \left\{-2\left[O_{\Phi}\left(z_{l}\right)\right]^{\beta}\right\}\right)
$$



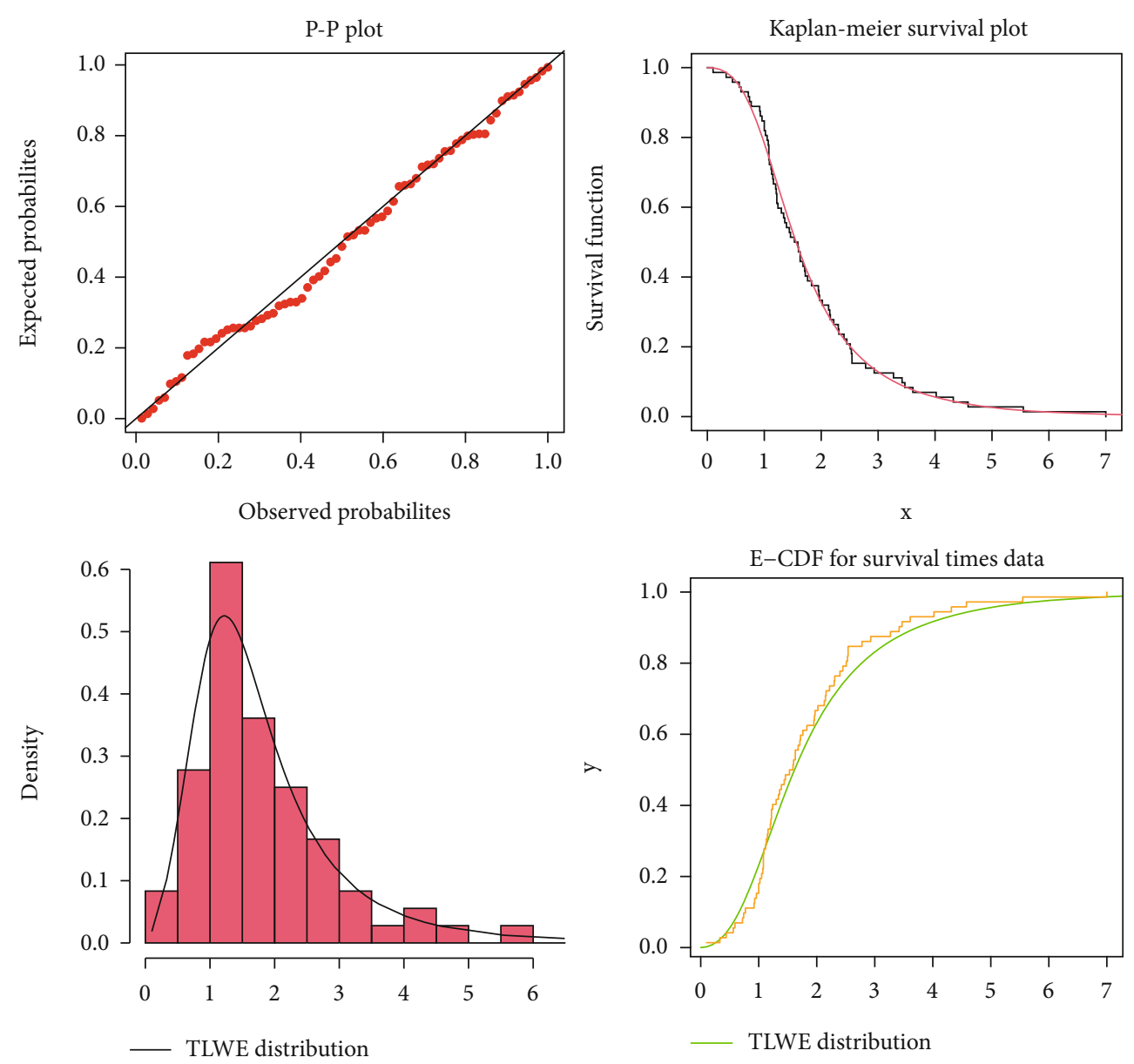

FIgURe 8: P-P, KMS plot, FPDF, and FCDF for the $2^{\text {nd }}$ data.

$$
\begin{aligned}
\frac{\partial L_{n}(\underline{P})}{\partial \beta}= & \frac{n}{\beta} \sum_{l=1}^{n} \log G_{\underline{\Phi}}\left(z_{l}\right)-\sum_{l=1}^{n} \log \bar{G}_{\underline{\Phi}}\left(z_{l}\right) \\
& -2 \sum_{l=1}^{n}\left[O_{\underline{\Phi}}\left(z_{l}\right)\right]^{\beta} \log \left[O_{\underline{\Phi}}\left(z_{l}\right)\right] \\
& +2(\alpha-1) \sum_{l=1}^{n} \frac{\log \left[O_{\underline{\Phi}}\left(z_{l}\right)\right]\left[O_{\underline{\Phi}}\left(z_{l}\right)\right]^{\beta} \exp \left\{-2\left[O_{\underline{\Phi}}\left(z_{l}\right)\right]^{\beta}\right\}}{1-\exp \left\{-2\left[O_{\Phi}\left(z_{l}\right)\right]^{\beta}\right\}}, \\
\frac{\partial L_{n}(\underline{P})}{\partial \underline{\Phi}}= & \sum_{l=1}^{n} \frac{\partial G_{\underline{\Phi}}\left(z_{l}\right) / \partial \underline{\Phi_{k}}}{G_{\Phi}\left(z_{l}\right)}+(\beta-1) \sum_{l=1}^{n} \frac{\partial G_{\underline{\Phi}}\left(z_{l}\right) / \partial \underline{\Phi}_{k}}{G_{\Phi}\left(z_{l}\right)} \\
& +(\beta+1) \sum_{l=1}^{n} \frac{\partial G_{\underline{\Phi}}\left(z_{l}\right) / \partial \underline{\Phi_{k}}}{\bar{G}_{\underline{\Phi}}\left(z_{l}\right)}-2 \sum_{l=1}^{n} O_{\underline{\Phi}}\left(z_{l}\right) \partial G_{\underline{\Phi}}\left(z_{l}\right) / \partial \underline{\Phi} k \\
& +2(\alpha-1) \sum_{l=1}^{n} \frac{\exp \left\{-2\left[O_{\Phi}\left(z_{l}\right)\right]^{\beta}\right\} O_{\Phi}\left(z_{l}\right) \partial G_{\Phi}\left(z_{l}\right) / \partial \underline{\Phi} k}{1-\exp \left\{-2\left[O_{\underline{\Phi}}\left(z_{l}\right)\right]^{\beta}\right\}},
\end{aligned}
$$

where $\underline{\delta}_{k}$ is the $k$ th member of the parameter vector $\delta$. The MLL estimation (MLLE) of $\underline{P}$ is achieved through solving the nonlinear equations $U_{n}(\underline{P})=0$.

\section{Graphical Simulations}

(i) We could perform numerical simulations to visually analyze the finite sample performance of the MLLEs utilizing biases (Bs) and mean squared errors (MSEs). For the assessment, the basic procedure had been used

(ii) Generate $N=1000$ samples of size $\left.n\right|_{(n=50,100, \cdots, 500)}$ from the TLWE model using (7)

(iii) Compute the MLLEs for $N=1000$ samples

(iv) Compute the standard errors (SErs) of the MLLEs for the 1000 samples

(v) Compute the Bs and MSErs given for $\underline{P}=\alpha, \beta, \theta$

The biases (left boxes) and MSEs (right windows) for the parameters are shown in Figures 3-5. The plots on the left demonstrate how the three biases grow with large sample $n$ , while the graphs on the right platform how the three MSEs change with $n$. The zero biases are depicted by the broken line as shown in Figure 1. From Figures 3-5, the biases with each parameter are typically negative and eventually drop to 0 as $n$ tends to infinity the MSEs with each parameter decrease to 0 as tends to infinity. 


\section{Modelling}

Inside this part, we look at two real-world data sets to show how adaptable the TLWE model is. The first data set (1.1, $1.4,1.3,1.7,1.9,1.8,1.6,2.2,1.7,2.7,4.1,1.8,1.5,1.2,1.4$, $3,1.7,2.3,1.6$, and 2) (see [25]) is known as the "failure times data," and it comprises lifetime data on "relief times" (in minutes) of analgesic-using individuals. In the second data set, [26] investigated and reported the "survival times" in days for 72 guinea pigs infected with virulent tubercle bacilli $(0.1,0.33,0.44,0.56,0.59,0.72,0.74,0.77,0.92,0.93$, $0.96,1,1,1.02,1.05,1.07,07,1.08,1.08,1.08,1.09,1.12$, $1.13,1.15,1.16,1.2,1.21,1.22,1.22,1.24,1.3,1.34,1.36$, $1.39,1.44,1.46,1.53,1.59,1.6,1.63,1.63,1.68,1.71,1.72$, $1.76,1.83,1.95,1.96,1.97,2.02,2.13,2.15,2.16,2.22,2.3$, $2.31,2.4,2.45,2.51,2.53,2.54,2.54,2.78,2.93,3.27,3.42$, $3.47,3.61,4.02,4.32,4.58$, and 5.55). Figure 6 shows the total time in the test (TTT) plot for determining the form of the empirical HRFs (first row). To explore the extreme observations, the box plot is sketched in Figure 6 (second row). To ensure that the normality state is maintained, the Q-Q plot is reported in Figure 6 (third row). Kernel density estimation (KDE) may be used to investigate the initial form of realworld data, and it is seen within Figure 6 (fourth row). According to Figure 6 (first row), we note that the HRF is "asymmetric monotonically increasing" for the two data sets. Based on Figure 6 (second row), we note that some extreme observations were spotted. Based on Figure 6 (third row), we see that the normality does not exist. Based on Figure 6 (fourth row), it is noted that the nonparametric Kernel densities are asymmetric.

We will compare the TLWE distribution's fits to various competing models, particularly exponential (E), odd Lindley E (OLE), Marshall-Olkin (MO) E (MOE), Moment E (MomE), the logarithmic Burr-Hatke E (LBHE), generalized MOE (GMOE), beta E (BE), MO Kumaraswamy E (MOKwE), Kumaraswamy E (KwE), and Kumaraswamy MOE (KwMOE). See the PDFs of the competitive models in $[27,28]$.

We discuss the Anderson-Darling $\left(C_{1}\right)$ and the CramérVon Mises $\left(C_{2}\right)$ statistics, as well as the KolmogorovSmirnov (D1) statistic as well as its associated $P$ value (D2). Moreover, we consider some other goodness-of-fit measures including the Akaike-Information-Criterion (IC) $\left(C_{3}\right)$, Bayesian IC $\left(C_{4}\right)$, Consistent-Akaike-IC $\left(C_{5}\right)$, and Hannan-Quinn IC $\left(C_{6}\right)$; Table 2 gives the MLLEs, SEs, and confidence interval (C.I.) values for the relief time data. Table 3 gives the MLLEs, SErs, and C.I. values for the survival time data. Table 4 illustrates the $C_{1}, C_{2}, C_{3}, C_{4}, C_{5}$, $C_{6}, \mathrm{D} 1$, and $\mathrm{D} 2$ for the relief time data. Table 5 refers to the $C_{1}, C_{2}, C_{3}, C_{4}, C_{5}, C_{6}, \mathrm{D} 1$, and D2 for the survival time data. Figure 7 gives the P-P plot, Kaplan-Meier survival (KMS) plot, fitted PDF (FPDF), and FCDF for the $1^{\text {st }}$ data. Figure 8 offers the P-P plot, KMS plot, fitted PDF (FPDF), and FCDF for the $2^{\text {nd }}$ data.

The TLWE model is much better than many common competitive models such as the exponential (standard version), MOE, OLE, LBHE, MomE, GMOE, KwE, MOKwE, and KwMOE models. As a result, the new lifespan model offers a practical alternate to all these models. For both data sets, we can see from Figures 7 and 8 that the TLWE model fits the two real data sets well.

\section{Discussion and Concluding Remarks}

We described and explored the TLWG family, a novel generator of continuous lifespan distributions, in this work. Statistical attributes of the family are offered, such as density function expansion, moments, incomplete moments, mean deviation, and Bonferroni and Lorenz curves. The new HRF might be described as "monotonically rising," "bathtub," "J shape," or "monotonically declining." FGM and MFGM families and CCO are often used to describe and visualize Copula of the basic kind. Regarding estimating model parameters, we glance at the MLL methodology. We conducted simulated studies to examine the limited sample behavior of MLL estimations utilizing graphs, biases, and mean squared errors. Two applications to actual data sets demonstrate the relevance and versatility of the intended family.

As a future work, we can apply many new useful goodness-of-fit tests for right-censored validation such as the Nikulin-Rao-Robson goodness-of-fit test, modified Nikulin-Rao-Robson goodness-of-fit test, BagdonaviciusNikulin goodness-of-fit test, and modified BagdonaviciusNikulin goodness-of-fit test. However, some bivariate versions could be studied in more details.

\section{Data Availability}

If you would like to get the quantitative data set utilized to conduct the investigation described in the publication, please notify the person author.

\section{Conflicts of Interest}

The authors declare no conflict of interest.

\section{Acknowledgments}

The authors extend their appreciation to the Deanship of Scientific Research at Imam Mohammad Ibn Saud Islamic University for funding this work through Research Group no. RG-21-09-08.

\section{References}

[1] H. M. Yousof, A. Z. Afify, M. Alizadeh, N. S. Butt, G. G. Hamedani, and M. M. Ali, "The transmuted exponentiated generalized-G family of distributions," Pakistan Journal of Statistics and Operation Research, vol. 11, no. 4, pp. 441-464, 2015.

[2] M. Bourguignon, R. B. Silva, and G. M. Cordeiro, "The Weibull-G family of probability distributions," Journal of Data Science, vol. 12, no. 1, pp. 53-68, 2014.

[3] H. M. Yousof, A. Z. Afify, G. G. Hamedani, and G. Aryal, "The Burr X generator of distributions for lifetime data," Journal of Statistical Theory and Applications, vol. 16, no. 3, pp. 1-19, 2017. 
[4] A. H. Soliman, M. Elgarhy, and M. Shakil, "Type II half logistic family of distributions with applications," Pakistan Journal of Statistics and Operation Research, vol. 13, no. 2, pp. 245-264, 2017.

[5] F. Merovci, M. Alizadeh, H. M. Yousof, and G. G. Hamedani, "The exponentiated transmuted-G family of distributions: theory and applications," Communications in Statistics-Theory and Methods, vol. 46, no. 21, pp. 10800-10822, 2017.

[6] M. El-Morshedy, F. S. Alshammari, Y. S. Hamed, M. S. Eliwa, and H. M. Yousof, "A new family of continuous probability distributions," Entropy, vol. 23, no. 2, p. 194, 2021.

[7] H. M. Yousof, M. Rasekhi, A. Z. Afify, M. Alizadeh, I. Ghosh, and G. G. Hamedani, "The beta Weibull-G family of distributions: theory, characterizations and applications," Pakistan Journal of Statistics, vol. 33, pp. 95-116, 2017.

[8] M. C. Korkmaz, M. Alizadeh, H. M. Yousof, and N. S. Butt, "The generalized odd Weibull generated family of distributions: statistical properties and applications," Pakistan Journal of Statistics and Operation Research, vol. 14, no. 3, pp. 541556, 2018.

[9] M. Alizadeh, M. Rasekhi, H. M. Yousof, and G. G. Hamedani, "The transmuted Weibull G family of distributions," Hacettepe Journal of Mathematics and Statistics, vol. 47, no. 6, pp. 1-20, 2017.

[10] H. M. Yousof, M. Majumder, S. M. A. Jahanshahi, M. Masoom Ali, and G. G. Hamedani, "A new Weibull class of distributions: theory, characterizations and applications," Journal of Statistical Research of Iran, vol. 15, no. 1, pp. 45-82, 2018.

[11] S. Rezaei, B. B. Sadr, M. Alizadeh, and S. Nadarajah, “ToppLeone generated family of distributions: properties and applications," Communications in Statistics-Theory and Methods, vol. 46, no. 6, pp. 2893-2909, 2017.

[12] C. Chesneau and H. M. Yousof, "On a special generalized mixture class of probabilistic models," Journal of Nonlinear Modeling and Analysis, vol. 3, no. 1, pp. 71-92, 2021.

[13] A. A. Al-Babtain, I. Elbatal, C. Chesneau, and M. Elgarhy, "Sine Topp-Leone-G family of distributions: theory and applications," Open Physics, vol. 18, no. 1, pp. 574-593, 2020.

[14] R. A. Bantan, F. Jamal, C. Chesneau, and M. Elgarhy, “Type II power Topp-Leone generated family of distributions with statistical inference and applications," Symmetry, vol. 12, no. 1, 2020.

[15] R. A. Bantan, F. Jamal, C. Chesneau, and M. Elgarhy, "A new power Topp-Leone generated family of distributions with applications," Entropy, vol. 21, no. 12, 2019.

[16] A. S. Hassan, M. Elgarhy, and Z. Ahmad, "Type II generalized Topp-Leone family of distributions: properties and applications," Journal of Data Science, vol. 17, no. 4, pp. 638-659, 2019.

[17] D. J. G. Farlie, "The performance of some correlation coefficients for a general bivariate distribution," Biometrika, vol. 47, no. 3-4, pp. 307-323, 1960.

[18] E. J. Gumbel, "Bivariate logistic distributions," Journal of the American Statistical Association, vol. 56, no. 294, pp. 335349, 1961.

[19] E. J. Gumbel, "Bivariate exponential distributions," Journal of the American Statistical Association, vol. 55, no. 292, pp. 698-707, 1960.

[20] D. Morgenstern, "Einfache beispiele zweidimensionaler verteilungen," Mitteilingsblatt fur Mathematische Statistik, vol. 8, pp. 234-235, 1956.
[21] J. A. Rodriguez-Lallena and M. Ubeda-Flores, "A new class of bivariate copulas," Statistics and Probability Letters, vol. 66, no. 3, pp. 315-325, 2004.

[22] N. Balakrishna and C. D. Lai, "Construction of bivariate distributions," Springer Science \& Business Media, 2009.

[23] E. S. A. El-Sherpieny, H. Z. Muhammed, and E. M. Almetwally, "Bivariate Weibull-G family based on copula function: properties, Bayesian and non-Bayesian estimation and applications," Statistics, Optimization \& Information Computing, vol. $\mathrm{x}, 31$ pages, 2021.

[24] E. S. A. El-Sherpieny, E. M. Almetwally, and H. Z. Muhammed, "Bayesian and non-bayesian estimation for the parameter of bivariate generalized Rayleigh distribution based on Clayton copula under progressive type-II censoring with random removal," Sankhya A, pp. 1-38, 2021.

[25] T. Bjerkedal, "Acquisition of resistance in guinea pigs infected with different doses of virulent tubercle bacilli," American Journal of Hygiene, vol. 72, pp. 130-148, 1960.

[26] J. Gross and V. A. Clark, "Survival distributions: reliability applications in the biometrical sciences," John Wiley, New York, USA, 1975.

[27] H. Elgohari, M. Ibrahim, and H. M. Yousof, "A new probability distribution for modeling failure and service times: properties, copulas and various estimation methods," Statistics, Optimization \& Information Computing, vol. 9, no. 3, pp. 555-586, 2021.

[28] M. Ibrahim, E. A. EA, and H. M. Yousof, "A new distribution for modeling lifetime data with different methods of estimation and censored regression modeling," Statistics, Optimization \& Information Computing, vol. 8, no. 2, pp. 610-630, 2020. 